\title{
Avaliação ecológica rápida da fauna de vespas (Hymenoptera: Aculeata) do Parque Nacional da Serra do Divisor, Acre, Brasil
}

\author{
Elder F. MORATO ${ }^{1}$, Sérvio Túlio AMARANTE², Orlando Tobias SILVEIRA ${ }^{3}$
}

\begin{abstract}
RESUMO
O Parque Nacional da Serra do Divisor (PNSD), localizado a noroeste do Estado do Acre, é considerado uma das áreas de maior diversidade da Amazônia. A importância de se considerar insetos em programas de conservação tem sido muito enfatizada. Vespas solitárias e sociais são componentes muito importantes dos ecossistemas, devido à posição que ocupam nas redes alimentares. Esse trabalho teve como objetivo realizar uma avaliação ecológica rápida da fauna de vespas do PNSD, para subsidiar a elaboração de um plano de manejo do referido parque. A amostragem foi realizada em 12 sítios de coleta, situados no interior de oito tipologias de vegetação. Os insetos foram coletados através de armadilhas Malaise, as quais foram expostas em todos os sítios por 24 horas, totalizando 288 horas de amostragem. Foram consideradas nas análises vespas das famílias Chalcididae, Eucharitidae, Evaniidae, Mutillidae, Pompilidae, Crabronidae e Vespidae. Ao todo, foram coletados 366 indivíduos distribuídos em 40 gêneros e 85 espécies. Os gêneros Ephuta (Mutillidae), Trypoxylon (Crabronidae) e Conura (Chalcididae) foram os mais ricos em espécies. Os sítios localizados na região norte do PNSD, considerada zona intangível e zona primitiva, foram os mais ricos em espécies. Cerca de $65 \%$ das espécies foram exclusivas a um único sítio, o que significa que as amostras possuem pequena similaridade faunística. Algumas espécies coletadas são consideradas raras.
\end{abstract}

PalaVraS-ChaVe: Vespas, Hymenoptera, Acre, Diversidade, Parque Nacional da Serra do Divisor.

\section{Rapid ecological assessment of wasp fauna (Hymenoptera: Aculeata) of the Serra do Divisor National Park, Acre, Brazil}

\begin{abstract}
The Serra do Divisor National Park (PNSD), located at the northwest of Acre State, Amazonia, is considered an area of great biodiversity. The question of considering insects in conservation programs. Solitary and social wasps are important components of the terrestrial ecosystems due to their position in trophic webs. The present study aimed at making a rapid ecological assessment of the wasps from the PNSD in order to support the elaboration of a conservation and management plan for that park. The insects were sampled in 12 sites located in eight forest types by Malaise traps that operated in each for 24 hours, totaling 288 hours of sampling. The results on the families Chalcididae, Eucharitidae, Evaniidae, Mutillidae, Pompilidae, Crabronidae and Vespidae are presented here. On the whole, 366 wasps were collected representing 40 genera and 85 species. The genera Ephuta (Mutillidae), Trypoxylon (Crabronidae) and Conura (Chalcididae) were the most specious. The sites situated at the northern region of the PNSD, the intangible and primitive zones, were the most species rich. Some collected species were considered rare and about $65 \%$ of species were exclusive to only one site. This means that the samples have little faunal similarity.
\end{abstract}

KEYWORDS: Wasps, Hymenoptera, Acre, Diversity, Serra do Divisor National Park.

\footnotetext{
1 Universidade Federal do Acre, Centro de Ciências Biológicas e da Natureza, Distrito Industrial, Rio Branco, AC. 69915-900. E-mail: elderfmorato@yahoo.com.br

${ }^{2}$ Museu de Zoologia - Universidade de São Paulo. Av. Nazaré, 481, Ipiranga, São Paulo, SP. 04263-000. E-mail: serviopa@usp.br

${ }^{3}$ Museu Paraense Emílio Goeldi. Av. Perimetral, 1901, Terra Firme, Belém, PA. 66017-970. E-mail: orlando@museu-goeldi.br
} 


\section{INTRODUÇÃO}

O estado do Acre situado na Amazônia sul-ocidental possui apenas 9\% do seu território desmatado (Salimon \& Brown, 2000). Apesar de possuir várias unidades de conservação (Acre, 1991), sua biota é muito pouco conhecida, uma vez que poucos inventários foram realizados. Contudo, estudos têm demonstrado a existência no Estado de áreas com grande biodiversidade e elevado grau de endemismo (e.g., Prance, 1973; Brown, 1991; Haffer, 1987; Brown, 1977; Silveira et al., 1997; Calouro, 1999; Vaz-de-Mello, 1999; Whittaker \& Oren, 1999; Guilherme, 2001; Morato, 2001; Souza et al., 2003). Novas espécies de Hymenoptera vêm sendo descritas (Silveira \& Carpenter, 1995; Azevedo \& Batista, 2002; Azevedo, Helmer \& Morato, 2002; Pedro \& Camargo, 2003; Moure, 2003; Oliveira \& Nemésio, 2003). Uma dessas áreas é o Parque Nacional da Serra do Divisor (PNSD), localizado a noroeste do estado.

Uma Avaliação Ecológica Rápida (AER) é um método diagnóstico usado para inferir sobre o estado de conservação de ecossistemas naturais terrestres (Nascimento \& Viana, 1999) ou aquáticos (Corgosinho et al., 2004) e baseia-se no emprego de indicadores biológicos e/ou biofísicos. O principal objetivo de uma REA é a redução do tempo e do custo para a avaliação do estado de conservação de áreas naturais de interesse, sem a necessidade de inventários detalhados.

Os programas de conservação, de um modo geral, não têm contemplado os invertebrados, principalmente os insetos (Dourojeanni, 1990), apesar de sua grande diversidade, sobretudo nas florestas tropicais (Erwin, 1982; Wilson, 1987) e de sua importância funcional nos ecossistemas terrestres (Price, 1984; Didham et al., 1996).

Contudo, autores têm chamado a atenção para a relevância de se considerar os insetos e outros invertebrados em programas de conservação, devido ao seu valor intrínseco e sua importância nos ecossistemas, bem como no monitoramento ambiental, seja como bioindicadores de qualidade ambiental ou de padrōes de diversidade em outros grupos (e.g., Dourojeanni, 1990; Brown, 1991; Samways, 1994; New, 1995; Morato \& Campos, 2000).

De interesse especial para a conservação são os insetos da ordem Hymenoptera, em especial as vespas, sejam sociais, solitárias ou parasitóides. Esses grupos incluem insetos que possuem grande variação em estrutura, fisiologia e comportamento (LaSalle \& Gauld, 1993). A pressão de predação e parasitismo que exercem nos ecossistemas é muito grande e deve representar importante mecanismo de regulação das populações das espécies de presas, de modo a permitir uma grande diversidade (LaSalle \& Gauld, 1993; O’Neill, 2001; Cirelli \& Penteado-Dias, 2003).
Suas populaçóes possuem tamanho efetivo e diversidade genética menores do que a de outros insetos, o que as tornam mais sensíveis às perturbações ambientais e propensas a extinçōes (LaSalle \& Gauld, 1993). Sob esse aspecto, também poderiam ser usados como bioindicadores de alteraçôes ambientais (e.g., Beyer, Miller \& Fleming, 1987; Tscharntke, Gathmann, Steefan-Dewenter, 1998; Morato \& Campos, 2000; Azevedo, Helmer \& Morato, 2002).

Há mais de 34.000 espécies de vespas aculeadas descritas no mundo todo. Destas, a maioria $(90 \%)$ é solitária (Evans \& Eberhard, 1970). Cerca de 14.700 espécies são das famílias Pompilidae, Crabronidae, Sphecidae e Vespidae (subfamília Eumeninae). As três primeiras abrangem cerca de 65\% das espécies de vespas aculeadas (Martins \& Pimenta, 1993).

Amarante (2002; 2005) listou 1.858 espécies de Crabronidae e Sphecidae para a região Neotropical. Destas, 645 ocorrem no Brasil, mas apenas 40 foram listadas para o Acre, contrastando com as 212 registradas para o Pará e as 153 para o Amazonas. É importante lembrar que a maior parte desses registros, para esses dois estados amazônicos, provêm de localidades ao longo dos principais rios dessa região, predominantemente, no Amazonas e Solimões. Além disso, os registros para o Acre são recentes, em grande parte reportados por Amarante (2002). É, portanto, evidente que ainda pouco se conhece sobre essas duas famílias para as regiōes mais afastadas dos rios Solimões e Amazonas, onde se inclui o Acre.

A maioria das 102 morfoespécies de Bethylidae coletadas pelo primeiro autor deste trabalho (E.F.M.) durante uma avaliação ecológica rápida no Parque Nacional da Serra do Divisor é, provavelmente, composta por espécies ainda não descritas (Azevedo, 1999; Azevedo, Helmer \& Morato, 2002). Destas, 13 pertencem ao gênero Apenesia, das quais 10 são novas (Azevedo \& Batista, 2002). Esses dados, somados aos acima mencionados, indicam que deve existir uma grande diversidade de vespas no Brasil e, em particular, no Acre.

Os Mutillidae constituem uma família cosmopolita de vespas que apresentam pronunciado polimorfismo entre os machos e fêmeas (Borror, Triplehorn $\&$ Johnson, 1992). As últimas são ápteras e parasitam larvas e pupas de várias espécies de vespas, abelhas, besouros e moscas. Embora o grupo seja especioso, muito pouco se sabe sobre as espécies brasileiras (Morato, 1994; Garcia, Cambra \& Melo, 2006).

Já as vespas parasíticas reúnem mais de 130.000 espécies descritas (Goulet \& Huber, 1993). Os Evaniidae contribuem com 400 espécies, os Chalcididae com 1.743 e os Eucharitidae com 413 (Goulet \& Huber, 1993; Heraty, 2002; Delvare \& Arias-Penna, 2006).

Armadilha Malaise (Steyskal, 1981) constitue um dos principais métodos empregados para a amostragem de vespas 
solitárias e sociais (Menke \& Parker, 1996; Kumagai, 2002; Silveira, 2002; Cirelli \& Penteado-Dias, 2003), tanto em estudos de curta (Azevedo, Helmer \& Morato, 2002) como de longa duração (Shlyakhtenok \& Agunovich, 2001; Strazanac et al., 2003).

Este trabalho teve como objetivo realizar uma avaliação ecológica rápida da fauna de vespas do PNSD, para subsidiar a elaboração de um plano de manejo do referido parque. Mais especificamente pretende avaliar a riqueza de vespas (Chalcididae, Eucharitidae, Evaniidae, Mutillidae, Pompilidae, Crabronidae e Vespidae) de diferentes tipos de vegetação do PNSD a partir de uma avaliação ecológica rápida.

\section{MATERIAL E MÉTODOS}

As amostragens foram realizadas no Parque Nacional da Serra do Divisor (PNSD), localizado a noroeste do Estado do Acre, entre as coordenadas $74^{\circ} 00^{\prime}$ e $72^{\circ} 45^{\prime} \mathrm{W}$ e $7{ }^{\circ} 15^{\prime}$ e $9^{\circ} 04^{\prime}$ S. O PNSD possui uma superfície de cerca de 843.000 ha, incluindo parte dos municípios de Cruzeiro do Sul, Mâncio Lima, Rodrigues Alves, Porto Walter e Marechal Thaumaturgo.

O clima na região é quente e úmido, com temperatura média anual igual a $24,2^{\circ} \mathrm{C}$, máxima média anual igual a $37^{\circ}$ e mínima média anual igual a $5^{\circ} \mathrm{C}$. A umidade relativa do ar varia entre 80 e $90 \%$ e a precipitação entre 1.750 e $2.000 \mathrm{~mm}$ anuais. Os meses menos chuvosos são junho, julho e agosto (Brasil, 1977).

O relevo da maior parte do PNSD é colinoso com altitudes variando entre 200 e $300 \mathrm{~m}$, desenvolvido sobre argilitos da formação Solimões de idade plio-pleistocênica e também argilitos vermelhos da formação Ramon de idade terciária. $\mathrm{Na}$ extremidade oeste ocorre uma zona de montanhas cuja altitude pode chegar a $580 \mathrm{~m}$ (Brasil, 1977).
Os principais rios do PNSD são o Juruá, Juruá Mirim, Ouro Preto, das Minas, Moa e Azul, ao longo dos quais foram registradas mais de 500 famílias humanas.

A maior parte da região possui vegetação do tipo floresta ombrófila aberta de palmeiras, de bambus e de cipós. Aproximadamente um terço da área possui floresta ombrófila densa: floresta sub-montana e floresta densa dos baixos platôs (Brasil, 1977).

A amostragem foi realizada em 12 sítios de coleta, os quais ficavam situados no interior de 8 diferentes tipologias de vegetação (Associação S.O.S. Amazônia, 1998; Silveira, 2005; Acre, 2006). Os nomes das tipologias e localização dos sítios são mostrados na tabela 1 .

Devido a grande extensão do PNSD, a amostragem foi dividida em duas etapas. A primeira ocorreu nas tipologias do Setor Norte do parque no mês de novembro de 1996. A segunda ocorreu no Setor Sul e foi realizada em março de 1997.

As vespas foram coletadas por meio de uma armadilha Malaise, a qual coleta insetos por interceptação em vôo e permaneceu por 24 horas em todos os sítios, totalizando 288 horas de amostragem. O material coletado foi depositado na Coleção Entomológica do Departamento de Ciências da Natureza (UFAC, AC), Museu de Zoologia (USP, SP) e Museu Paraense Emílio Goeldi (MPEG - PA).

Valores de correlação entre abundância e riqueza de vespas e riqueza florística das áreas amostrais (Associação S.O.S. Amazônia, 1998) e riqueza de vespas foram calculados através do coeficiente de correlação não paramétrico de Spearman (Siegel \& Castellan, 1988).

A similaridade entre os sítios amostrais, em relação à composição de espécies, foi calculada através do índice qualitativo de Jaccard (Ludwig \& Reynolds, 1988). O método

Tabela 1 - Características dos sítios amostrais do Parque Nacional da Serra do Divisor, Acre. N = região norte do PNSD; S = região sul.

\begin{tabular}{|c|c|c|}
\hline Sítio & Tipologia & Coordenadas \\
\hline N1 & Floresta densa sub-montana & $7^{0} 26^{\prime} 36^{\prime \prime}$ S; 73040'28" W \\
\hline N3 & Floresta densa sub-montana & $7^{0} 28^{\prime} 38^{\prime \prime} \mathrm{S} ; 73^{\circ} 41^{\prime} 54^{\prime \prime} \mathrm{W}$ \\
\hline N5 & Floresta densa nos platôs & $7^{\circ} 27^{\prime} 07^{\prime \prime}$ s; 734'06" W \\
\hline N6 & Floresta aberta de palmeiras em depósitos coluviais & $7^{0} 26^{\prime} 27^{\prime \prime}$ S; 73039'28” W \\
\hline N8 & Floresta aberta de palmeiras nos platôs & 7033'24" S; 73016'35" W \\
\hline N9 & Floresta de várzea & 7027'02" S; 73036'30" W \\
\hline S1 & $\begin{array}{l}\text { Floresta aberta de palmeiras com bambu dominante sobre a formação Solimões do } \\
\text { Pleistoceno }\end{array}$ & $8^{0} 16^{\prime} 51^{\prime \prime} \mathrm{S} ; 73^{0} 15^{\prime} 13^{\prime \prime} \mathrm{W}$ \\
\hline S4 & Floresta aberta de palmeiras sobre a formação Solimões do Pleistoceno & $8^{0} 24^{\prime} 19^{\prime \prime}$ S; 72051'28” W \\
\hline S6 & Floresta aberta de palmeiras nos terraços altos & 8033’30" S; 72053’30" W \\
\hline S8 & Vegetação de "baboca" em floresta aberta de palmeiras nos terraços altos & $8^{\circ} 33^{\prime} 50^{\prime \prime}$ S; $72^{\circ} 51^{\prime} 45^{\prime \prime} \mathrm{W}$ \\
\hline S10 & Floresta aberta de palmeiras com bambu dominante sobre a formação Solimões & $8^{0} 52^{\prime} 31^{\prime \prime}$ S; $72^{\circ} 46^{\prime} 55^{\prime \prime} \mathrm{W}$ \\
\hline S11 & Floresta de várzea & 8051'37" S; 72052'20" W \\
\hline
\end{tabular}


da rarefação foi empregado para comparar o número esperado de espécies dos sítios em função do tamanho amostral (Ludwig \& Reynolds, 1988).

\section{RESULTADOS}

Foram coletados 366 exemplares de 40 gêneros e 84 espécies nos diferentes sítios do PNSD (tabela 2). As famílias mais representativas em termos de abundância individual foram Evaniidae (44\%), Vespidae (21,6\%) e Mutillidae (14,5\%). As mais representativas em espécies foram Crabronidae (25\%), Vespidae (23,8\%) e Mutillidae (17,9\%). Os gêneros mais ricos em espécies foram Ephuta (Mutillidae) (15,5\%), Trypoxylon (Crabronidae) (10,7\%) e Conura (Chalcididae) $(7,1 \%)$. Evaniella sp. e Semaeomyia sp. (Evaniidae) foram responsáveis por cerca de 39\% dos indivíduos coletados. A grande maioria $(97,6 \%)$ das espécies foi representada por 20 ou menos indivíduos.

Do total de espécies, 57 (68\%) foram coletadas exclusivamente em um único sítio amostral. Contudo, algumas espécies foram coletadas na maioria dos sítios. Evaniella sp. e Semaeomyia sp. (Evaniidae) ocorreram em 92\% e $83 \%$ dos sítios, respectivamente; Ephuta flavidens (Mutillidae) e Angiopolybia pallens (Vespidae) em 75\%.

Os sítios que apresentaram maior riqueza foram N6 (S $=25)$ e $S 6(S=24)$. Houve uma correlação positiva entre abundância de indivíduos coletados nos sítios e a riqueza $\left(\mathrm{r}_{\mathrm{s}}\right.$ $=0,92$; g.l. $=11 ; \mathrm{p}=0,000019)$ e entre a riqueza e o número de espécies exclusivas $\left(r_{s}=0,85 ;\right.$ g.l. $\left.=11 ; \mathrm{p}=0,000499\right)$.

De modo geral, os sítios localizados na região norte do PNSD apresentaram valores maiores de abundância, riqueza e espécies exclusivas (Figura 1). A curva de riqueza esperada de espécies $(E(S))$ da região norte em função do tamanho amostral obtida por rarefação foi superior à obtida para a região sul (Figura 2).

Os valores de similaridade entre os sítios em relação à composição em espécies foram de modo geral muito baixos e inferiores a $40 \%$. Os sítios da região sul foram mais similares entre si (similaridade média $=22 \%$ ) do que com os da região norte (similaridade média $=16,2 \%$ ) e os da regiāo norte entre si $($ similaridade média $=16,1 \%)$.

Tabela 2 - Abundância e riqueza em espécies de vespas sociais, solitárias e parasitóides coletadas em diferentes sítios do Parque Nacional da Serra do Divisor, Acre. $\mathrm{N}=$ região norte do PNSD; $\mathrm{S}=$ região sul.

\begin{tabular}{|c|c|c|c|c|c|c|c|c|c|c|c|c|c|}
\hline Espécies & $\mathrm{N} 1$ & N3 & N5 & N6 & N8 & N9 & $\mathrm{S} 1$ & S4 & S6 & S8 & $\mathrm{S} 10$ & $\mathrm{~S} 11$ & TOTAL \\
\hline \multicolumn{14}{|l|}{ Chalcididae } \\
\hline Aspirrhina sp. & & & 1 & & & & & & & & & & 1 \\
\hline Brachymeria sp. & & 1 & 1 & 2 & & & & & & & 1 & & 5 \\
\hline Conura sp.1 & & & & & 1 & & & & & & & & 1 \\
\hline Conura sp.2 & & & & & & & & & 1 & & & & 1 \\
\hline Conura sp.3 & & & 1 & & & & & & & & & & 1 \\
\hline Conura sp.4 & & & & & & & & 1 & & & & & 1 \\
\hline Conura sp.5 & 1 & & & & & & & & & & 1 & & 2 \\
\hline Conura sp.6 & & & & & & 2 & & & & & & & 2 \\
\hline Melanosmicra sp. & & & & & 2 & & & & & & & & 2 \\
\hline Notaspidium sp. & & & & & & 2 & & & & & & & 2 \\
\hline Stypiura sp.1 & 1 & & 1 & & & & & & & & & & 2 \\
\hline Stypiura sp.2 & & & & & 1 & & & & & & & & 1 \\
\hline \multicolumn{14}{|l|}{ Eucharitidae } \\
\hline Kapala sp. & & 1 & & 1 & & 1 & & & & & & & 3 \\
\hline Lophyrocera sp. & & 1 & & 2 & & & & & 1 & & & & 4 \\
\hline Obeza sp. & & & & & & & & & & 1 & & & 1 \\
\hline \multicolumn{14}{|l|}{ Evaniidae } \\
\hline Evaniella sp. & 1 & 2 & 10 & 9 & 7 & 14 & 5 & 4 & 9 & & 5 & 4 & 70 \\
\hline Semaeomyia sp. & & 1 & 6 & 7 & & 19 & 3 & 5 & 23 & 1 & 6 & 2 & 73 \\
\hline Hyptia sp. & & & 2 & 2 & 1 & 3 & & & 4 & & 6 & & 18 \\
\hline \multicolumn{14}{|l|}{ Mutillidae } \\
\hline Ephuta (Ephuta) argentula (D. Torre) & & & & & & 6 & & & 1 & & & & 7 \\
\hline
\end{tabular}


Tabela 2 - Continuação

\begin{tabular}{|c|c|c|c|c|c|c|c|c|c|c|c|c|c|}
\hline Espécies & N1 & N3 & N5 & N6 & N8 & N9 & $\mathrm{S} 1$ & S4 & S6 & S8 & $\mathrm{S} 10$ & $\mathrm{~S} 11$ & TOTAL \\
\hline E. (E.) flavidens Mickel & & 1 & 1 & 5 & 1 & 4 & 1 & & 1 & & 1 & 1 & 16 \\
\hline E. (E.) fugax (Smith) & & & & & & & & & 1 & & & & 1 \\
\hline E. (E.) trifida (Gerst.) & & & & & & 1 & & & & & & & 1 \\
\hline E. (E.) aff. perfica & & & 1 & & & & & & & & 1 & & 2 \\
\hline E. (E.) aff. scutellata & & & & & & & & & & & 2 & & 2 \\
\hline E. (E.) sp. 1 & & & & & & & & & & & & & 1 \\
\hline E. (E.) sp.2 & & & & & & 1 & & & & & 2 & & 3 \\
\hline E. (E.) sp.3 & & & & & & & & & 2 & & & & 2 \\
\hline E. (E.) sp.4 & & & & & & & & & 1 & & & & 1 \\
\hline E. (E.) sp.5 & & & & & & & & & 1 & & & & 1 \\
\hline E. (Ephutopsis) sp. & & & & & & 1 & & & 2 & & & 1 & 4 \\
\hline E. (Ephuseabra) sp. & & & & 1 & & & & & & & & & 1 \\
\hline Pseudomethoca sp. & & & 1 & 1 & & 2 & 1 & & 1 & & 1 & & 7 \\
\hline Traumatomutilla pomba (Cresson) & & 1 & & 3 & & & & & & & & & 4 \\
\hline \multicolumn{14}{|l|}{ Pompilidae } \\
\hline Ageniellini sp. & & & 1 & & & 1 & & & & & & & 2 \\
\hline Aporus sp. & & & & & & & & & 1 & & & & 1 \\
\hline Auplopus sp. & & 1 & & & & & & & & & & & 1 \\
\hline Ceropales cfr. cubensis & & & & & & & 1 & & 1 & & & & 2 \\
\hline Irenangelus furtivus Evans & & 1 & & & & & & & & & 1 & & 2 \\
\hline Notocyphus sp.1 & & & & & & & & & 1 & & & & 1 \\
\hline Notocyphus sp.2 & & & & & 1 & & & & & & & & 1 \\
\hline Priochilus nubilus Banks & & & & 1 & & & & & & & & & 1 \\
\hline P. splendidulum (Fabricius) & & & & & & 2 & & & & & & & 2 \\
\hline Priocnemella sp. & & & & & & & & & 1 & & & & 1 \\
\hline \multicolumn{14}{|l|}{ Crabronidae } \\
\hline Anacrabro fritzi Leclercq & & & 1 & & & & & & & & & & 1 \\
\hline Enoplolindenius nisera Leclercq & & & & & & 1 & & & & & & & 1 \\
\hline Liris sp.1 & & 1 & 1 & & & & & & & & & & 2 \\
\hline Liris sp.2 & & 1 & & & & & & & & & & & 1 \\
\hline Liris sp.3 & & & & & & & 1 & & 1 & & & & 2 \\
\hline Liris sp.4 & & & 1 & & & & & & & & & & 1 \\
\hline Lyroda sp. & & & & 2 & & & & & & & & & 2 \\
\hline Metanysson sp.1 & & & & & & 1 & & & & & & & 1 \\
\hline Metanysson sp.2 & & & & 1 & & & & & & & 1 & & 2 \\
\hline Pison cameroni Kohl & & & 1 & & & & & & & & & & 1 \\
\hline Quexua verticallis (F. Smith) & & & 3 & & & & & & & & & & 3 \\
\hline Trachypus caenosus Rubio & & & & & & & & & 1 & & & & 1 \\
\hline Trypoxylon (Trypargilum) sp.1 & & & 1 & & & & & & & & & & 1 \\
\hline Trypoxylon (Trypoxylon) sp.1 & & & & & & & & & & & & 1 & 1 \\
\hline Trypoxylon (Trypoxylon) sp.2 & & & & 1 & & & & & & & & & 1 \\
\hline Trypoxylon (Trypoxylon) sp.3 & & & & 1 & & & & & & & & & 1 \\
\hline Trypoxylon (Trypoxylon) sp.4 & & & 1 & & & & & & 1 & & & & 2 \\
\hline Trypoxylon (Trypoxylon) sp.5 & & & & 1 & & & 1 & & & & & & 2 \\
\hline Trypoxylon (Trypoxylon) sp.6 & & & & 1 & & & & & & & & & 1 \\
\hline
\end{tabular}


Tabela 2 - Continuação

\begin{tabular}{|c|c|c|c|c|c|c|c|c|c|c|c|c|c|}
\hline Espécies & N1 & N3 & N5 & N6 & N8 & N9 & $\mathrm{S} 1$ & S4 & S6 & S8 & $\mathrm{S} 10$ & $\mathrm{~S} 11$ & TOTAL \\
\hline Trypoxylon (Trypoxylon) sp.7 & & & & 1 & & & 1 & & & & & & 2 \\
\hline Trypoxylon (Trypoxylon) sp.8 & & 1 & & & & & & & & & & & 1 \\
\hline \multicolumn{14}{|l|}{ Vespidae } \\
\hline Agelaia angulata (Fabricius) & & & & 1 & & & & & & & & & 1 \\
\hline A. fulvofasciata (Degeer) & 6 & 2 & 4 & 1 & 1 & & 2 & & & & & & 16 \\
\hline A. Iobipleura Richards & & 1 & & & & & & & & & & & 1 \\
\hline A. myrmecophila (Ducke) & & 2 & & & & & & & & & & & 2 \\
\hline Angiopolybia pallens (Lepeletier) & & 1 & 1 & 6 & 1 & 3 & & 2 & 3 & 1 & 1 & 1 & 20 \\
\hline A. paraensis (Spinola) & & 1 & & & & 1 & & & & & & & 2 \\
\hline Brachygastra albula Richards & 1 & & & & & & & & & & & & 1 \\
\hline B. lecheguana (Latreille) & 1 & & & & & & & & & & & & 1 \\
\hline B. moebiana (Saussure) & 2 & & & & & & & & & & & & 2 \\
\hline Myschocyttarus duckei (du Buysson) & & & & 1 & & & & & & & & & 1 \\
\hline M. labiatus (Fabricius) & 1 & & & & & & & & & & & & 1 \\
\hline Myschocyttarus grupo heliconius sp. & 1 & & & & & & & & & & & & 1 \\
\hline Myschocyttarus grupo artifex sp. & & & & 1 & & & & & & & & & 1 \\
\hline Polybia catillifex Moebius & & & & & & & 1 & & & & & & 1 \\
\hline P. dimorpha Richards & & & & 6 & & & & & 2 & & & & 8 \\
\hline P. emaciata Lucas & & & & & & 3 & & & & & & & 3 \\
\hline P. rejecta (Fabricius) & 11 & & & & & & & & & & & & 11 \\
\hline P. tinctipennis Fox & 2 & & & & & & & & & & & & 2 \\
\hline Protopolybia chartergoides (Gribodo) & & 3 & & & & & & & & & & & 3 \\
\hline Synoeca virginea (Fabricius) & & & & & & & & & 1 & & & & 1 \\
\hline Abundância & 28 & 23 & 40 & 59 & 16 & 68 & 17 & 12 & 61 & 3 & 29 & 10 & 366 \\
\hline № de espécies & 11 & 18 & 20 & 25 & 9 & 19 & 10 & 4 & 24 & 3 & 13 & 6 & 85 \\
\hline $\mathrm{N}^{0}$ de espécies exclusivas & 7 & 6 & 7 & 10 & 4 & 7 & 1 & 1 & 11 & 1 & 1 & 1 & 57 \\
\hline
\end{tabular}

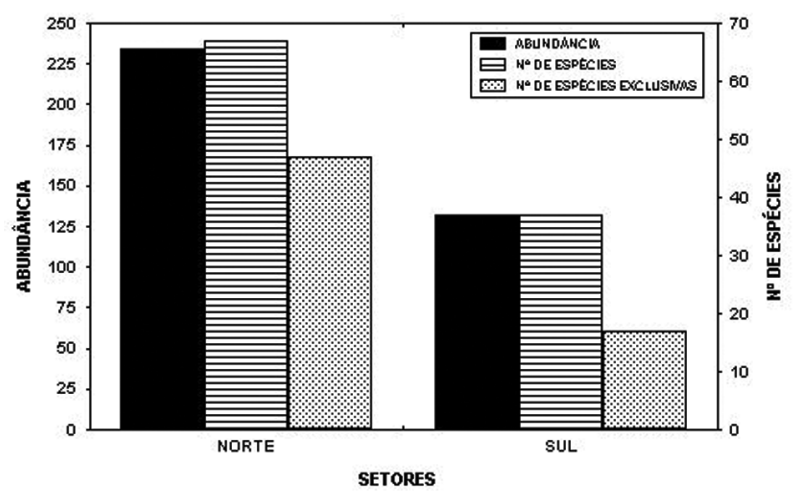

Figura 1 - Abundância e riqueza em espécies de vespas dos setores norte e sul do Parque Nacional da Serra do Divisor, Acre.

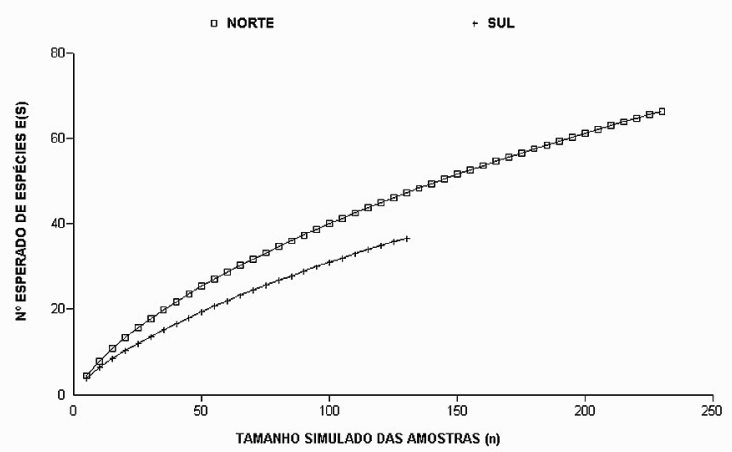

Figura 2 - Curvas de rarefação para a riqueza esperada de espécies $(E(S))$ de vespas dos setores norte e sul do Parque Nacional da Serra do Divisor, Acre. 
Não houve correlação significativa entre riqueza florística e riqueza de vespas $\left(r_{\mathrm{s}}=0,41 ; \mathrm{g} .1 \mathrm{l}=6 ; \mathrm{p}=0,3552\right)$, considerando para isso apenas 7 sítios onde foram feitos levantamentos florísticos concomitantemente à amostragem de vespas.

\section{DISCUSSÃO}

Não foi possível denominar muitas espécies pertencentes à maioria das famílias aqui consideradas. Isto é conseqüência do conhecimento insuficiente e fragmentado da fauna de Hymenoptera da Amazônia Ocidental, em particular de Aculeata e Parasitica. Por exemplo, a maioria das 102 morfoespécies de Bethylidae, coletadas durante a mesma avaliação ecológica rápida no $\mathrm{PNSD}$, deve ser desconhecida para a ciência (Azevedo, 1999; Azevedo, Helmer \& Morato, 2002). Destas, 13 são espécies do gênero Apenesia, das quais 10 são novas (Azevedo \& Batista, 2002).

Mais da metade das espécies foi coletada exclusivamente em um único sítio, o que sugere que as amostras possuem pequena similaridade faunística. Os sítios da região norte, de modo geral, apresentaram maior riqueza em espécies do que os da região sul. Resultado semelhante foi também encontrado para os Bethylidae amostrados por armadilhas Malaise no PNSD (Azevedo, Helmer \& Morato, 2002). É possível que isso se deva ao fato de ser a região norte do PNSD menos desmatada que a região sul (Associação S.O.S. Amazônia, 1998).

Das 20 espécies de vespas eussociais coletadas, Angiopolybia pallens (Lepeletier) repete o padrão de maior freqüência observado por Silveira (2002) e Silveira et al. (2004) em Caxiuanã, Pará. Trata-se certamente da espécie mais comum de vespa social em florestas amazônicas. Por outro lado, Agelaia lobipleura Richards, Polybia dimorpha Richards, P. tinctipennis Fox, P. catillifex Moebius, Brachygastra albula Richards e B. moebiana (Saussure) são relativamente mais raras em coleçōes. As demais espécies, principalmente de Agelaia e Angiopolybia são bastante comuns em coletas com armadilhas. Em Caxiuanã, numa área pouco menor que as dos sítios amostrados no PNSD, Norte e Sul, respectivamente, mas com esforço de captura cerca de 60 vezes maior, foram capturadas 23 espécies de 6 gêneros em armadilhas Malaise. Notavelmente, estes números não são muito diferentes dos totais obtidos nos sítios de coleta da parte Norte do PNSD (18 espécies e 6 gêneros). Entretanto, apenas a contribuição exclusiva do sítio N1 (8 espécies) já perfaz excepcionais 40\% do total das espécies coletadas no PNSD inteiro. O fato de este local ter sido parcialmente queimado (por um raio) antes das coletas (Associação S.O.S. Amazônia, 1998) talvez explique a maior riqueza, em particular a presença de três espécies de Brachygastra, pois o gênero normalmente apresenta algumas espécies que se encontram em vegetação aberta ou pioneira (Richards, 1978).
Nos sítios da parte Sul do PNSD, por outro lado, o número de espécies capturadas foi bem menor, apenas cinco de quatro gêneros, sendo este resultado mais condizente com o baixo tempo de exposição das armadilhas em cada sítio.

Armadilhas de intercepção (Malaise, armadilha suspensa) são relativamente ineficientes quando comparadas à procura ativa por ninhos e indivíduos em vôo (ver Silveira, 2002). Em Caxiuanã, o número de espécies capturadas em armadilhas não ultrapassou $30 \%$ de um total de 79 espécies encontradas naquela região. Isto indica que armadilhas Malaise amostram apenas uma fração menor da fauna de vespas sociais num dado local e que, portanto, não devem permitir em geral comparaçôes muito significativas de diversidade entre sítios. Entretanto, é interessante notar em respeito aos grupos de vespas solitárias coletados no PNSD, que os valores de riqueza de espécies por sítio (agrupadas as várias famílias) não são correlacionados àqueles verificados para as vespas sociais.

Apesar de vespas da família Mutillidae serem consideradas raras (Schmidt \& Buchmann, 1986) elas foram bem representadas nas coletas em termos de espécies e indivíduos.

A riqueza de espécies de Crabronidae reflete o esperado ao apresentar um maior número de espécies em Trypoxylon e Liris, respectivamente, o segundo e o quinto gêneros em números de espécies para a Região Neotropical. Resultados similares, com a predominância de espécies desses dois gêneros, foram encontrados pelo segundo autor (inf. pessoal) em uma recente série de amostragens padronizadas em diversas localidades da Mata Atlântica. O conhecimento da fauna desses dois gêneros para a regiáo Neotropical é, ainda, bastante incompleto e heterogêneo, a Amazônia Ocidental destacando-se como uma área onde tal conhecimento é particularmente insuficiente, consequiência de uma amostragem historicamente precária e dispersa. Não surpreende, portanto, que todas as espécies desses dois gêneros não puderam ser determinadas, tanto porque podem ser novas quanto por ser extremamente difícil determinar com segurança espécies desses gêneros provenientes de várias áreas da Região Neotropical. Entretanto, é importante salientar que é muito mais provável que sejam desconhecidas para a ciência, pois o número de espécies conhecidas para a Região Neotropical está bem abaixo do que presume-se existir de fato.

\section{AGRADECIMENTOS}

À Fundação S.O.S. Amazônia, Rio Branco, Acre, pela oportunidade e apoio financeiro dos trabalhos de campo. Á Dra. Verônica Telma da Rocha Passos (Universidade Federal do Acre) pelo auxílio na elaboração dos dados descritivos da área de estudo e apoio logístico. Aos Drs. Diomedes Quintero e Roberto A. Cambra pela identificação das vespas das famílias Mutillidae e Evaniidae. 


\section{BIBLIOGRAFIA CITADA}

Acre. 1991. Atlas Geográfico Ambiental do Acre. Secretaria de Meio Ambiente, Instituto de Meio Ambiente do Acre, Rio Branco, Acre. 48pp.

Acre. 2006. Zoneamento Ecológico-Econômico. Fase II. Documento Sintese. Escala 1:250.000. Governo do Estado do Acre, Secretaria de Estado de Meio Ambiente e Recursos Naturais, Rio Branco, Acre. 354pp.

Amarante, S.T.P. 2002. A synonymic catalog of the Neotropical Crabronidae and Sphecidae (Hymenoptera: Apoidea). Arquivos de Zoologia, 37(1): 1-139.

Amarante, S.T.P. 2005. Addendum and corrections to a synonymic catalog of Crabronidae and Sphecidae. Papéis Avulsos de Zoologia, 45(1): 1-18.

Associação S.O.S. Amazônia. 1998. Plano de Manejo, Parque Nacional da Serra do Divisor, Acre, Brasil. Rio Branco, S.O.S. Amazônia, IBAMA, The Nature Conservancy. 532pp.

Azevedo, C.O. 1999. On the Neotropical Rhabdepyris Kieffer (Hymenoptera, Bethylidae) of the sub-genus Chlorepyris. Revista Brasileira de Zoologia, 16(3): 887-897.

Azevedo, C.O.; Batista, M.L. 2002. New species of Apenesia (Hymenoptera, Bethylidae) from the Parque Nacional da Serra do Divisor, Acre, Brazil. Revista Brasileira de Entomologia, 46(1): 25-32.

Azevedo, C.O.; Helmer J.L.; Morato, E.F. 2002. Diversidade de Bethylidae (Hymenoptera) do Parque Nacional da Serra do Divisor, Acre, Brasil e seu uso no plano de manejo e conservação da área. Acta Amazonica, 32(1): 71-82.

Beyer, W.N.; Miller, G.W,; Fleming, W.J. 1987. Populations of trap-nesting wasps near a major source of fluoride emissions in western Tennessee. Proceedings of the Entomological Society of Washington, 89(3): 478-482.

Borror, D.J.; Triplehorn, C.A.; Johnson, N.F. 1992. An introduction to the study of insects. Ged. Saunders College Publishing, Fort Worth. 875pp.

Brasil. 1977. Projeto RADAMBRASIL: levantamento de recursos naturais. Vol.13. Folhas SB/SC. 18. Javari/Contamana. Departamento Nacional da Produção Mineral, Rio de Janeiro. 413pp.

Brown, K.S., Jr. 1977. Centros de evolução, refúgios quaternários e conservação de patrimônios genéticos na região neotropical: padrôes de diferenciação em Ithomiinae (Lepidoptera: Nymphalidae). Acta Amazonica, 7(1): 75-137.

Brown, K.S. Jr. 1991. Conservation of Neotropical environments: insects as indicators. In: Collins, N.M.; Thomas, J.A. (Eds). The Conservation of Insects and Their Habitats. Academic Press, London. p. 349-404.

Calouro, A.M. 1999. Riqueza de mamíferos de grande e médio porte do Parque Nacional da Serra do Divisor (Acre, Brasil). Revista Brasileira de Zoologia, 16(Supl.2): 195-213.

Cirelli, K.R.N.; Penteado-Dias, A.M. 2003. Análise da riqueza da fauna de Braconidae (Hymenoptera, Ichneumonidae) em remanescentes naturais da Área de Proteção Ambiental (APA) de Descalvado, SP. Revista Brasileira de Entomologia, 47(1): 89-98.

Corgosinho, P.H.C.; Calixto, L.S.F.; Fernandes, P.L.; Gagliardi, L.M.; Balsamão, V.L.P. 2004. Diversidade de habitats e padrões de diversidade e abundância dos bentos ao longo de um afluente do reservatório de Três Marias, MG. Arquivos do Instituto Biológico, São Paulo, 71(2): 227-232.

Delvare. G.; Arias-Penna, D.C. 2006. Família Chalcididae. In: Fernández, F.; Sharkey, M.J. (Eds.). Introducción a los Hymenoptera de la Región Neotropical. Sociedad Colombiana de Entomologia y Universidad Nacional de Colombia, Bogotá. p. 647-660.

Didham, R.K.; Ghazoul, J.; Stork, N.E.; Davis, A.J. 1996. Insects in fragmented forests: a functional approach. TREE, 11(6): 255-259.

Dourojeanni, M.J. 1990. Entomology and biodiversity conservation in Latin America. American Entomologist, 36(2): 88-93.

Erwin, T.L. 1982. Tropical forests: their richness in Coleoptera and other arthropod species. The Coleopterists Bulletin, 36(1): 74-75.

Evans, H.E.; Eberhard, M.J.W. 1970. The Wasps. The University of Michigan Press, Ann Arbor. 265pp.

Garcia, E.Q.; Cambra, R.; Melo, G.A.R. 2006. Sexual associations of two species of mutillid wasps (Hymenoptera, Mutillidae), with the description of a new species of Anomophotopsis. Revista Brasileira de Entomologia, 50(3): 379-384.

Goulet, H.; Huber, J. T. 1993. Hymenoptera of the world: an identification guide of families. Research Branch, Agriculture Canada, Otawa. 668pp.

Guilherme, E. 2001. Comunidade de aves do Campus e Parque Zoobotânico da Universidade Federal do Acre, Brasil. Tangara, 1(2): 57-73.

Haffer, J. 1987. Biogeography of neotropical birds. In: Whitmore, T.C.; Prance, G.T. (Eds). Biogeography and Quaternary History in Tropical America. Clarendon Press, Oxford. p.105-150.

Heraty, J.M. 2002. A revision of the genera of Eucharitidae (Hymenoptera: Chalcididae) of the world. Memoirs of the Entomological Institute, 68: 367pp.

Kumagai, A.F. 2002. Os Ichneumonidae (Hymenoptera) da Estação Ecológica da universidade Federal de Minas Gerais, Belo Horizonte, com ênfase nas espécies de Pimplinae. Revista Brasileira de Entomologia, 46(2): 189-194.

LaSalle, J.; Gauld, I.D. 1993. Hymenoptera: their diversity, and their impact on the diversity of other organisms. In: LaSalle, J.; Gauld, I.D. (Eds). Hymenoptera and Biodiversity. C.A.B. International, Wallingford. p.1-26.

Ludwig, J.A.; Reynolds, J.F. 1988. Statistical ecology: a primer on methods and computing. John Wiley \& Sons, New York. 337pp.

Martins, R.P.; Pimenta, H.R. 1993. Ecologia e comportamento de vespas solitárias predadoras. Ciência Hoje, 15(90): 14-19.

Menke, A.S.; Parker, F.D. 1996. Phenology of ammophiline wasps in a Premontane Wet Forest in Costa Rica (Hymenoptera, 
Sphecidae, Ammophilini). Journal of Hymenoptera Research, 5: 184-189.

Morato, E.F. 1994. Xystromutilla asperiventris André, 1905 (Mutillidae) reared from sphecid wasps in trap-nests, Manaus, Amazonas, Brazil. Sphecos, 28: 13-14.

Morato, E.F. 2001. Ocorrência de Aglae caerulea Lepeletier \& Serville (Hymenoptera, Apidae, Apini, Euglossina) no Estado do Acre, Brasil. Revista Brasileira de Zoologia, 18(3): 1031-1034.

Morato, E.F.; Campos, L.A.O. 2000. Efeitos da fragmentação florestal sobre vespas e abelhas solitárias em uma área da Amazônia Central. Revista Brasileira de Zoologia, 17(2): 429-444

Moure, J.S. 2003. Duas espécies novas de Centris (Heterocentris) Cockerell, da região amazônica e do Brasil Central (Hymenoptera, Apoidea). Revista Brasileira de Zoologia, 20(2): 265-268.

Nascimento, H.E.M.; Viana, V.M. 1999. Estrutura e dinâmica de eco-unidades em um fragmento de floresta estacional semidecidual na região de Piracicaba, SP. Scientia Forestalis, 55: 29-47.

New, T.R. 1995. Introduction to Invertebrate Conservation Biology. Oxford University Press, Oxford. 194pp.

Oliveira, M.L. de.; Nemésio, A. 2003. Exaerete lepeletieri (Hymenoptera: Apidae: Apini: Euglossina): a new cleptoparasitic bee from Amazônia. Lundiana, 4(2): 117-120.

O'Neill, K.M. 2001. Solitary Wasps: Behavior and Natural History. Cornell University Press, Ithaca. 406pp.

Pedro, S.R.M.; Camargo, J.M.F. de. 2003. Meliponini neotropicais: o gênero Partamona Schwarz, 1939 (Hymenoptera, Apidae). Revista Brasileira de Entomologia, 47(Supl.1):1-117.

Prance, G.T. 1973. Phytogeographic support for the theory of Pleistocene forest refuges in the Amazon basin based on evidence from distribution patterns in Caryocaraceae, Chrysobalanaceae, Dichapetalaceae and Lecythidaceae. Acta Amazonica, 3(3): 5-28.

Price, P. W. 1984. Insect Ecology. 2ed. John Wiley \& Sons, New York. 607pp.

Richards, O. W. 1978. The social wasps of the Americas, excluding the Vespinae. British Museum (Natural History), London. 580pp.

Salimon, C.I.; Brown, I.F. 2000. Secondary forest in western Amazonia: significant sinks for carbon released from deforestation ? Interciencia, 25(4): 198-202.

Samways, M.J. 1994. Insect Conservation Biology. Chapman \& Hall, London. 358pp.

Schmidt, J.O.; Buchmann, S.L. 1986. Are mutillids scarce? (Hymenoptera: Mutillidae). Pan-Pacific Entomologist, 62(2): 103-104.

Shlyakhtenok, A.S.; Agunovich, R.G. 2001. The dynamics of species composition and abundance of wasps from the families Pompilidae, Sphecidae and Vespidae (Hymenoptera: Aculeata) in sucessional pine biogeocenoses of the Berezinskii biosphere reserve. Russian Journal of Ecology, 32(2): 126-129.

Siegel, S.; Castellan, N.J., Jr. 1988. Nonparametric statistics for the behavioral sciences. 2ed. McGraw-Hill, New York. 399pp.
Silveira, M. 2005. A floresta aberta com bambu no sudoeste da Amazônia: padröes e processos em múltiplas escalas. Edufac, Rio Branco, Acre. 157pp.

Silveira, M.; de Paula, N.M.C.; Brown, I.F.; Borges, H.B.N.; Daly, D.; Ferreira, L.A. 1997. Os 'buracos negros' da diversidade. Ciência Hoje, 22(128): 64-65.

Silveira, O.T. 2002. Surveying neotropical social wasps. An evaluation of methods in the "Ferreira Penna Research Station (ECFPn), in Caxiuanã, PA, Brazil (Hym., Vespidae, Polistinae). Papéis Avulsos de Zoologia, São Paulo, 42(12): 299-323.

Silveira, O.T.; Carpenter, J.M. 1995. A new species of Agelaia Lepeletier from brazilian Amazonia (Hymenoptera: Vespidae: Polistinae). Journal of the New York Entomological Society, 103(1): 69-72.

Silveira, O. T.; Esposito, M. C.; Santos Jr, J. N.; Gemaque, F. E., Jr. 2004. Social wasps and bees captured in carrion traps in a rain forest in Brazil (Hymenoptera: Vespidae; Apidae). Entomological Science, 8: 33-39.

Souza, M.B. de; Silveira, M.; Lopes, M.R.M.; Vieira, L.J.S.; Guilherme, E.; Calouro, A.M.; Morato, E.F. 2003. A biodiversidade no Estado do Acre: conhecimento atual, conservação e perspectivas. T\& C Amazônia, 1(3): 45-56.

Steyskal, G.C. 1981. A bibliography of the malaise trap. Proceedings of the Entomological Society of Washington, 83(2): 225-229.

Strazanac, J.S.; Smith, D.R.; Braud, R.A.; Fritzler, C.J.; Butler, L. 2003. Symphyta (Hymenoptera) species richness in mixed oak-pine forests in the central Appalachians. Proceedings of the Entomological Society of Washington, 105(1): 131-137.

Tscharntke, T.; Gathmann, A.; Steefan-Dewenter, I. 1998. Bioindication using trap-nesting bees and wasps and their natural enemies: community structure and interactions. Journal of Applied Ecology, 35: 708-719.

Vaz-de-Mello, F.Z. 1999. Scarabaeidae s.str. (Coleoptera, Scarabaeoidea) de um fragmento de floresta amazônica no Estado do Acre, Brasil. 1. Taxocenose. Anais da Sociedade Entomológica do Brasil, 28(3): 447-453.

Whittaker, A.; Oren, D.C. 1999. Important ornithological records from the Rio Juruá, western Amazonian forest bird community. Bulletin of the British Ornithological Club, 119(4): 235-260.

Wilson, E.O. 1987. The arboreal ant fauna of Peruvian Amazon forests: a first assessment. Biotropica, 19(3): 245-251.

Recebido em 31/01/2007

Aceito em 29/05/2008 
